\title{
INTERPLANETARY SCATTERING OF FAST SOLAR ELECTRONS DEDUCED FROM TYPE III BURSTS OBSERVED AT LOW FREQUENCIES
}

\author{
H. ALVAREZ* \\ Radio Astronomy Observatory, University of Michigan, Ann Arbor, Mich. 48104, U.S.A. \\ and \\ R. P. LIN \\ Space Sciences Laboratory, University of California, Berkeley, Calif. 94720, U.S.A.
}

\begin{abstract}
Observations of low frequency solar type III radio bursts and the associated fast solar electrons show that the total path length traveled by the particles between the Sun and the Earth is significantly greater than the length of the smooth Archimedean spiral trajectory followed by the centroid of the type III exciter (Alvarez et al., 1975). Here we assume that the ratio of electron path length and the spiral length increases approximately as $r^{n}$, where $r$ is heliocentric distance, and then compute the radio bursts arrival time at $1 \mathrm{AU}$ for different values of $n$. A comparison with the radio observations indicates that the best fit occurs for $n=1.5 \pm 1.0$. We interpret these results in terms of the variation of electron scattering with heliocentric distance.
\end{abstract}

\section{Introduction}

Spacecraft observations of the last few years have provided strong evidence that streams of fast solar electrons of approximately 10 to $10^{2} \mathrm{keV}$ energies excite type III solar radio bursts (Alvarez et al., 1972; Frank and Gurnett, 1972; Lin et al., 1973; Alvarez et al., 1975). The electrons presumably interact with the ambient plasma exciting plasma waves which in turn couple into electromagnetic waves at the local plasma frequency and/or its second harmonic (see review by Smith, 1974). However, the mechanism is not well understood, and in particular, it is not known what fraction of the electron stream energy is converted into plasma waves. Furthermore there are no direct observations bearing on the characteristics of the propagation of the fast electrons between the Sun and the Earth orbit (1 $\mathrm{AU})$.

From the observations of type III bursts at low frequencies Fainberg et al. (1972) reported an apparent decrease of about a factor of two in the exciter stream velocity between the Sun and the Earth. Lin et al. (1973) suggested that pitch angle scattering of the electrons was primarily responsible for this apparent deceleration. This was supported by recent work (Alvarez et al., 1975, henceforth called Paper I) which showed that the observations are consistent with negligible energy loss of the fast electrons.

Using the same events analyzed in Paper I, this paper studies how the electron path length, $L$, increases with heliocentric distance, $r$. This is done by comparing the observation of radio burst arrival times at the Earth with those predicted from models. In these models we vary the rate of increase of path length with $r$. We

* Present address: Centro de Investigación de Astronomía, Apartado 264, Mérida, Venezuela. 
find that the radio observations can be explained by assuming that the effective path length traveled by the electrons increases rapidly with $r$. These results support the hypothesis that scattering of fast electrons in the interplanetary medium can produce apparent stream deceleration.

\section{The Analysis}

The four events analyzed are those of 16 May, 1971; 28 February, 1972; 27 March, 1972 and 19 August, 1972. These events were studied in detail in Paper I. The radio bursts were observed by the University of Michigan radiometer at 8 frequencies between $3.5 \mathrm{MHz}$ and $50 \mathrm{kHz}$; the associated fast solar electrons were observed by the University of California, Berkeley, detector at energies between 18 and $400 \mathrm{keV}$.

Some of the assumptions made in that study, relevant to the analysis presented here are: (a) the interplanetary magnetic field lines have the shape of an Archimedean spiral, i.e., the solar wind speed is constant; (b) regardless of their energy the fast electrons are ejected simultaneously from the Sun; (c) electrons of different energies travel the same total path length between the Sun and the Earth orbit; (d) the electrons do not lose significant energy; and (e) there is no radio wave scattering.

The analysis of the events onsets indicated that at $1 \mathrm{AU}$ the total path length $L$ of the electrons is longer than the spiral length $s$. The results that best fit the observations are shown in Table I where $K=L / s$ at $1 \mathrm{AU}$.

TABLE I

Electron path lengths between the Sun and $1 \mathrm{AU}$, from Alvarez et al. (1975)

\begin{tabular}{lcc}
\hline \multicolumn{1}{c}{ Event } & $s(\mathrm{AU})$ & $K=L / s$ \\
\hline 16 May 1971 & 1.14 & 1.70 \\
28 Feb. 1972 & 1.26 & 1.51 \\
27-28 March 1972 & 1.18 & 1.72 \\
19 August 1972 & 1.11 & 1.25 \\
\hline
\end{tabular}

To study the propagation of the fast electrons at intermediate distances between the Sun and $1 \mathrm{AU}$ we designate by $\rho_{n}$ the ratio of the electron effective path length $L(r)$ and the length of the Archimedean spiral, $s(r)$,

$$
\rho_{n}=\frac{L(r)}{s(r)}
$$

We assume that $\rho_{n}$ varies with $r$ as follows:

$$
\rho_{n}=1+(K-1)\left(\frac{r}{215}\right)^{n},
$$

where $r$ is in solar radii and $n \geqslant 0$. This expression was adopted because except for 


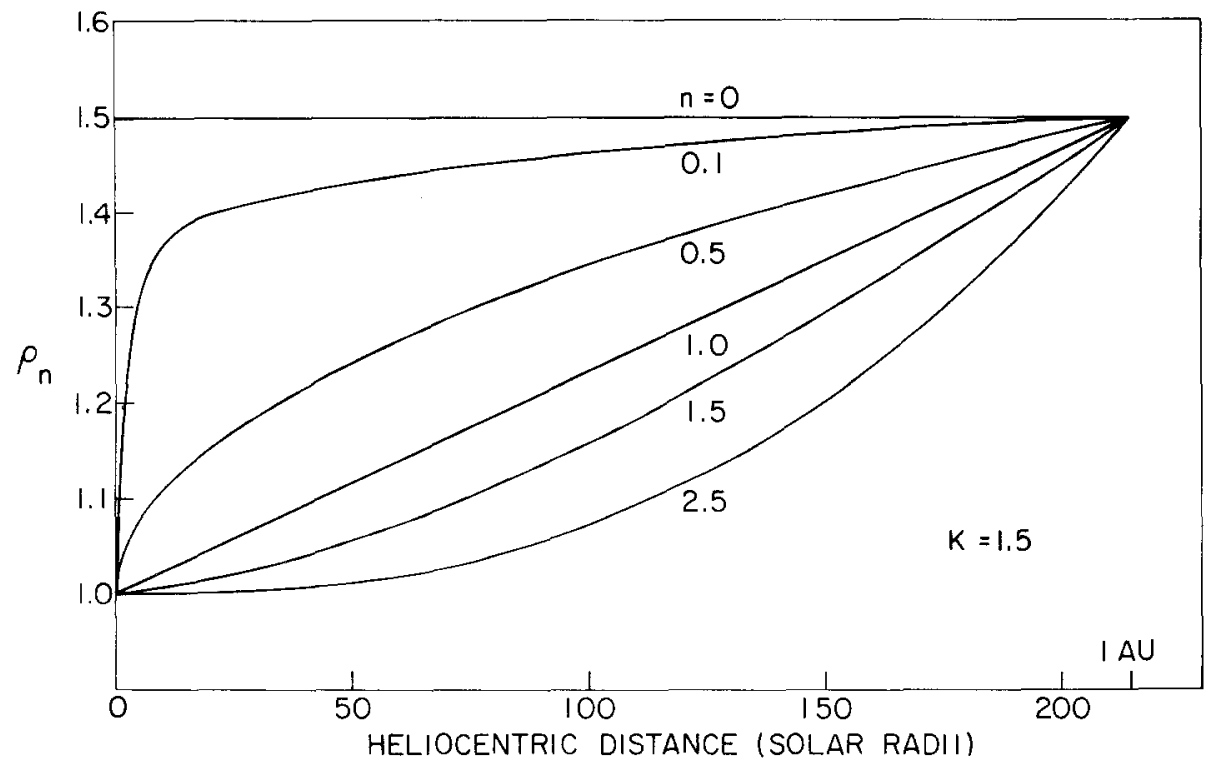

Fig. 1. Plots of $\rho_{n}$ as a function of $r$ for $K=1.5$ and different $n$. $\rho_{n}$ is the ratio of electrons total path length and the length of the Archimede's spiral that guides the stream centroid. The rate of increase of the total path length with heliocentric distance is varied by varying $n$.

$n=0$ it is monotonically increasing and because $\rho_{n}(0)=1$ and $\rho_{n}(215)=K$, otherwise it is arbitrary. Notice that $n=0$ corresponds to a uniform distribution of path length.

Figure 1 illustrates the function $\rho_{n}(r)$ computed for $K=1.5$. Increasing $n$ makes the scattering increase more rapidly with $r$.

Using the same models as in Paper I we have computed the arrival time of the radio waves at the Earth. The only difference is that now we assume that the total distance traveled by the fast electrons between the Sun and the plasma level at distance $r$ is $\rho_{n}(r) \times s(r)$ rather than $s(r)$. The computations were done for different values of $n$ and Figure 2 illustrates the comparison of the computed curves with the radio observations for the event of 28 February, 1972. The times of the peaks of the radio burst are denoted by horizontal segments. The burst onsets were not used because they could not be measured with the required accuracy. The times are expressed as delays referred to the onset of the $3.5 \mathrm{MHz}$ burst computed for fundamental emission. The time origin is at 1623.5 UT. The curves are computed for second harmonic radiation, and are constrained to pass through the middle of the $50 \mathrm{kHz}$ error bar. The observations are approximately bound by curves with $n=0.5$ and $n=2.5$ with the best fit occurring for 1.5 . The apparent velocity is $0.19 c$ ( $c=$ velocity of light in vacuum). Although there is a large uncertainty in the $50 \mathrm{kHz}$ burst arrival time, we found that passing the curves through a point at the beginning or the end of the $50 \mathrm{kHz}$ error bar changes the velocity only in $\pm 5 \%$. Similar results obtained for the other events are shown in Table II where $\beta$ is velocity in units of $c$. 
TABLE II

Best fit parameters

\begin{tabular}{lrl}
\hline \multicolumn{1}{c}{ Event } & \multicolumn{1}{c}{$n$} & \multicolumn{1}{c}{$\beta$} \\
\hline 16 May 1971 & $1.0-1.5$ & $0.27-0.28$ \\
28 Feb. 1972 & 1.5 & 0.19 \\
27-28 March 1972 & $0.5-2.0$ & $0.24-0.26$ \\
19 August 1972 & $0.0-2.5$ & $0.23-0.29$
\end{tabular}

Of the cases studied we choose the 28 February, 1972 event to illustrate the results for two reasons. First, it has the best radio data, second, it is the only event for which the $50 \mathrm{kHz}$ burst occurred at $1 \mathrm{AU}$ ( $25 \mathrm{kHz}$ local plasma frequency). This condition is important for the correctness of the fitting process illustrated in Figure 2.

For the 28 February event the maximum of the radio burst emission at $1 \mathrm{AU}$ corresponds to the predicted onset time of about $10 \mathrm{keV}$ electrons (obtained by extrapolating the velocity dispersion observed for greater than $18 \mathrm{keV}$ electrons). We assume here that the radio emission maxima observed for higher frequencies, i.e., emission from regions between the Sun and $1 \mathrm{AU}$, is also due to approximately $10 \mathrm{keV}$ electrons. Then the results obtained here can be interpreted in terms of the propagation of these particles.

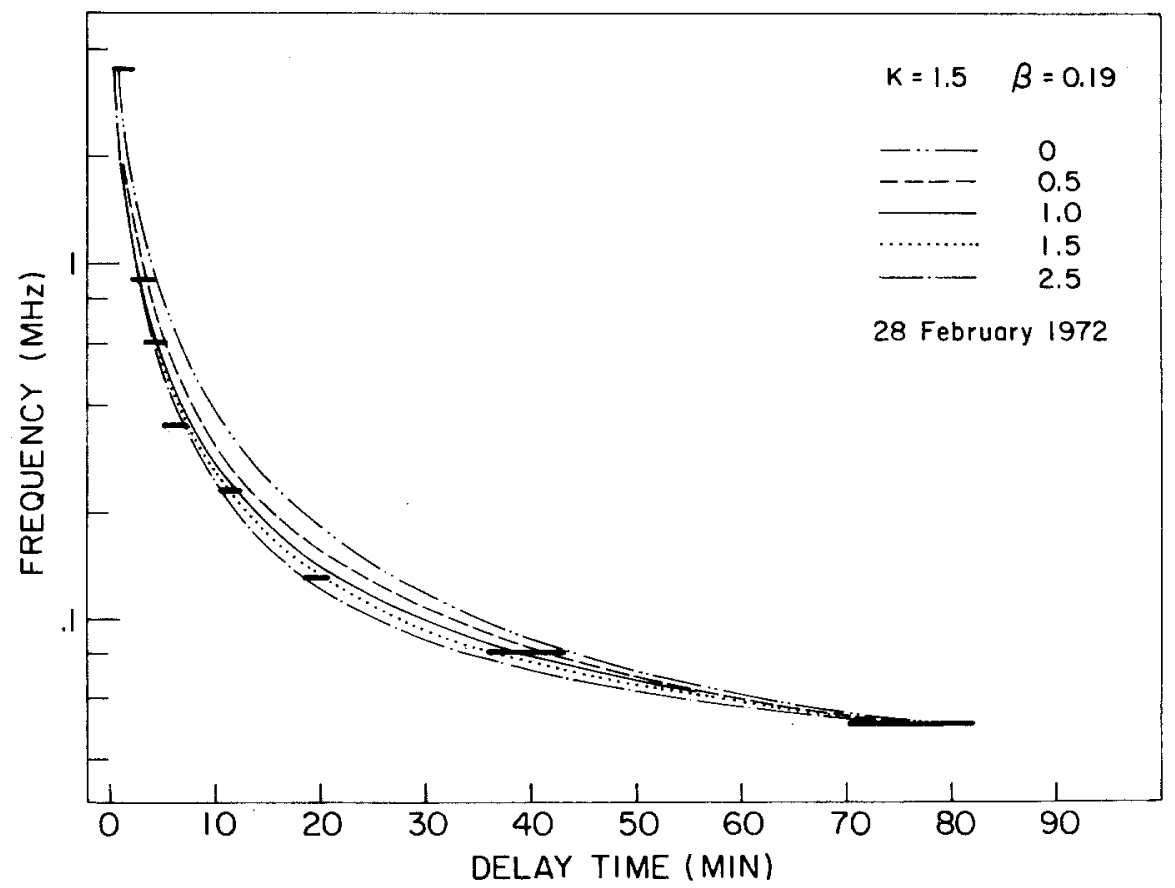

Fig. 2. Comparison of the observed and computed radio peak times for the 28 February 1972 event. The short delays observed at the upper frequencies can be obtained from the model by increasing $n$, while keeping constant the velocity of the electron stream. The same effect could be produced by decelerating electrons, however the results of this study do not support this hypothesis. 
Notice that increasing $n$ has the effect of increasing the frequency drift rate at high frequencies while decreasing it at low frequencies. This is the same effect that would be produced by a decelerating stream. $K$ in Equation 2 corresponds approximately to the apparent drop in velocity between the Sun and the Earth.

As the excess path length increases the electron flux versus time profiles show changes in shape which are consistent with increased scattering. The 19 August, 1972 event which is scatter-free has the smallest excess path length, while the 16 May, 1971 and 27-28 March, 1972 events are the most diffusive and have the largest path lengths (see Table I). Also if the scattering does not decrease rapidly with $r$ we would expect that $L(r) \approx s(r)$ close to the Sun since the bulk of the fast electrons would still have not been scattered. This is observed to be the case. We conclude that the excess path length is predominantly due to scattering rather than increased length of the Archimedean field line (due to wiggles which are "smooth" for the electron propagation).

\section{Acknowledgement}

One of us (H. A.) thanks Prof. F. T. Haddock for his support. The Radio work was done under NASA Grant NSG7004. The research at the University of California was supported in part by NASA Contract NAS5-11038.

\section{References}

Alvarez, H., Haddock, F. T., and Lin, R. P.: 1972, Solar Phys. 26, 468.

Alvarez, H., Lin, R. P., and Bame, S. J.: 1975, Solar Phys. 44, 485.

Fainberg, J., Evans, L. G., and Stone, R. G.: 1972, Science 178, 743

Frank, L. A. and Gurnett, D. A.: 1972, Solar Phys. 27, 446.

Lin, R. P., Evans, L. G., and Fainberg, J.: 1973, Astrophys. Lett. 14, 191.

Smith, D. F., 1974, Space Sci. Rev. 16, 91.

\section{Discussion}

R. Smith: To get the path length to $1 \mathrm{AU}$ don't you need particle spectral evolution?

Alvarez: No.

Lin: The radio observations permit us to follow the electrons inside $1 \mathrm{Au}$. We are assuming that the emission occurs at roughly the same time in the electron flux evolution at each distance.

Gurnett: Do the electron observations conform to diffusion models?

Lin: Diffusion models are not very sensitive to differences in the radial dependence of scattering irregularities.

Kellogg: What is a scatter free event?

Lin: That's when the flux of particles which have not scattered is observable above those that have scattered.

Melrose: Can you see the fluctuations which do the scattering?

Lin: There aren't many magnetic measurements with the time resolution needed to see these fluctuations. 